\title{
Insensitizing controls for the 1-d wave equation
}

\author{
René Dáger
}

\begin{abstract}
We study the insensitizing controllability property of the $1-d$ wave equation observed in some open set in two cases, when the control acts in an interior region and when it acts on the boundary. In both cases, when the control time is sufficiently large the $\epsilon$-insensitizing controllability holds. Moreover, for the boundary controlled equation the (exact) insensitizing controllability also holds.
\end{abstract}

\section{Introduction}

Consider the controlled wave equations with partially known initial data

$$
\begin{cases}y_{t t}-\Delta y=\xi+f \mathbf{1}_{\omega} & \text { in } \mathbb{R} \times(0,1), \\ y=0 & \text { in } \mathbb{R} \times\{0,1\} \\ y(0, x)=y^{0}+\tau_{0} z^{0} & \text { in }(0,1), \\ y_{t}(0, x)=y^{1}+\tau_{1} z^{1} & \text { in }(0,1),\end{cases}
$$

where $\omega$ is an open subset of $[0,1]$, and

$$
\begin{cases}y_{t t}-\Delta y=\xi & \text { in } \mathbb{R} \times(0,1), \\ y(t, 0)=g(t), \quad y(t, 0)=0 & \text { in } \mathbb{R}, \\ y(0, x)=y^{0}+\tau_{0} z^{0} & \text { in }(0,1), \\ y_{t}(0, x)=y^{1}+\tau_{1} z^{1} & \text { in }(0,1) .\end{cases}
$$

In both (1) and $(2) y^{0}, y^{1}$ and $\xi \in L^{2}(\mathbb{R} \times(0,1))$ are fixed functions. The last two equations in (1) or (2) indicate that the initial data are not exactly known. The terms $\tau_{0} z^{0}, \tau_{1} z^{1}$ with $\tau_{0}, \tau_{1} \in \mathbb{R}$ and $z^{0}, z^{1}$ satisfying

$$
\left\|z^{0}\right\|_{a}=\left\|z^{1}\right\|_{b}=1
$$

(in appropriate norms $\|\cdot\|_{a}$ and $\|\cdot\|_{b}$ guaranteeing the well-posedness of the corresponding equations) are small unknown perturbations of the original initial data $y^{0}, y^{1}$. Finally, $f \in L^{2}(\mathbb{R} \times$ $(0,1))$ and $g \in L^{2}(\mathbb{R})$ are control functions. The only difference between (1) and (2) is the location of the control, in equation (1) the control $f$ acts in an interior region $\omega$, while in (2) the control $g$ acts through a part of the boundary.

Let $\Phi$ be the functional defined on the solutions of the controlled equations (1) or (2) by

$$
\Phi(y)=\frac{1}{2} \int_{0}^{T} \int_{\mathcal{O}} y^{2} d x d t,
$$

where $T>0$ and $\mathcal{O}$ is an open subset of $[0,1]$. Note that $\Phi$ corresponds to observations of the solutions made in the interval $\mathcal{O}$ during a time interval of length $T$. 
Given a control $f$ (resp. $g$ ) we say that $f$ (resp. $g$ ) $\varepsilon$-insensitizes $\Phi$ if for every pair $z^{0}, z^{1}$ satisfying (3) the corresponding solution of (1) (resp. (2)) satisfies

$$
\left.\frac{d}{d \tau_{0}} \Phi(y)\right|_{\tau_{0}=\tau_{1}=0}=\left.\frac{d}{d \tau_{1}} \Phi(y)\right|_{\tau_{0}=\tau_{1}=0}=0 .
$$

The concept of insensitizing control was introduced by Lions in [9]. Later on, Bodart and Fabré in [3] proposed the weakened notion of $\varepsilon$-insensitizing control requiring

$$
\left.\left|\frac{d}{d \tau_{0}} \Phi(y)\right|_{\tau_{0}=\tau_{1}=0}|\leq \varepsilon, \quad| \frac{d}{d \tau_{1}} \Phi(y)\right|_{\tau_{0}=\tau_{1}=0} \mid \leq \varepsilon
$$

for $\varepsilon>0$.

In this paper we study the existence of insenstizing controls for systems (1) and (2). Our main results are (see theorems 7 and 13):

Let $\omega, \mathcal{O}$ be a non-empty open subset of $[0,1]$ and $T \geq 4$. Then,

- for any $\varepsilon>0$ there exist controls $\varepsilon$-insensitizing $\Phi$ along the solutions of (1).

- there exist controls insensitizing $\Phi$ along the solutions of (2).

To prove these results we use the technique proposed by Lions in [9]. For this concrete choice of $\Phi$, finding insensitizing controls is equivalent to finding controls driving the solutions of an auxiliary cascade system to rest, i.e., to a usual controllability problem, which is reduced to proving observability properties for the adjoint control-free cascade system. In particular, the existence of $\varepsilon$-insensitizing controls follow from a unique continuation property for the adjoint system. The main difficulty in this latter problem arises due to the non-standard coupling in the cascade system

We have to note that insenstizing or $\varepsilon$-insenstizing controllability have been generally studied in considerably more complex situations, namely, non-linear parabolic equations in $\mathbb{R}^{n}$ (see [14,6-11]). In this context our problem looks fairly simple. However, all those papers assume the observation and control regions to have non-empty intersection $(\omega \cap \mathcal{O} \neq \varnothing)$. In this sense, our equations provide the first example when $\varepsilon$-insensitizing controls exist for arbitrarily chosen observation and control regions. Up to now, the only known example of such situation was given in [10] for the $1-d$ linear heat equation with interior control and a particular choice of $\omega$ and $\mathcal{O}$. Besides, as far as we know, this is the first attempt to consider insenstizing controllability problems for the wave equation.

On the other hand, the existence of insensitizing controls for all the initial data for equation (2) is an interesting fact, since up to now, the insensitizing controls have been found as the limit of a sequence of $\varepsilon$-insensitizing controls for some particular choices of the initial data (see, e.g., $[1,2,7,8])$.

\section{Interior control}

In this section we concentrate ourselves in system (1). This is precisely the choice of control made in all the preceding works related to the insensitizing controllability of parabolic equations.

We assume $y^{0} \in H_{0}^{1}(0,1), y^{1} \in L^{2}(0,1)$. Then, system (1) has a unique solution of finite energy that satisfies, for every $T>0, y \in C\left([0, T], H_{0}^{1}(0,1)\right) \cap C^{1}\left([0, T], L^{2}(0,1)\right)$. In this case, 
the norms appearing in (3) are $\|\cdot\|_{a}=\|\cdot\|_{H_{0}^{1}(0,1)}$ and $\|\cdot\|_{b}=\|\cdot\|_{L^{2}(0,1)}$. In what follows to simplify the notations we denote

$$
\begin{gathered}
\|\cdot\|_{1}:=\|\cdot\|_{H_{0}^{1}(0,1)}, \quad\|\cdot\|_{0}:=\|\cdot\|_{L^{2}(0,1)}, \quad\|\cdot\|_{-1}:=\|\cdot\|_{H^{-1}(0,1)}, \\
\|\cdot\|_{1,0}:=\left(\|\cdot\|_{1}^{2}+\|\cdot\|_{0}^{2}\right)^{\frac{1}{2}}, \quad\|\cdot\|_{0,-1}:=\left(\|\cdot\|_{0}^{2}+\|\cdot\|_{-1}^{2}\right)^{\frac{1}{2}} .
\end{gathered}
$$

\subsection{Reduction to a unique continuation problem}

As stated above, the main technique for proving the existence of $\varepsilon$-insensitizing controls is to reduce the problem to proving a uniqueness result for the solutions of a cascade system. In this subsection we describe such reduction. These facts are well known in the context of parabolic equations, and we use here the same ideas. Although this scheme is rather standard, we include the details here since, as far as we know, they have never been written for the wave equation.

The first step is to reduce the problem to an approximate controllability problem. Consider the following controlled cascade system of wave equations

$$
\begin{gathered}
\begin{cases}\bar{y}_{t t}-\Delta \bar{y}=\xi+f \mathbf{1}_{\omega} & \text { in } \mathbb{R} \times(0,1), \\
\bar{y}=0 & \text { in } \mathbb{R} \times\{0,1\}, \\
\bar{y}(0, x)=y^{0}, \quad \bar{y}_{t}(0, x)=y^{1} & \text { in }(0,1),\end{cases} \\
\begin{cases}q_{t t}-\Delta q=\bar{y} \mathbf{1}_{\mathcal{O}} & \text { in } \mathbb{R} \times(0,1), \\
q=0 & \text { in } \mathbb{R} \times\{0,1\}, \\
q(T, x)=0, \quad q_{t}(T, x)=0 & \text { in }(0,1) .\end{cases}
\end{gathered}
$$

Note that, since $\bar{y} \mathbf{1}_{\mathcal{O}} \in L^{2}(\mathbb{R} \times(0,1))$, system (7) has a unique solution $q \in C\left([0, T], H_{0}^{1}(0,1)\right) \cap$ $C^{1}\left([0, T], L^{2}(0,1)\right)$. In particular, $\left(q(0,),. q_{t}(0,).\right) \in H_{0}^{1}(0,1) \times L^{2}(0,1)$.

It holds

Proposition 1 Given $y^{0} \in H_{0}^{1}(0,1), y^{1} \in L^{2}(0,1)$ and $\xi \in L^{2}(\mathbb{R} \times(0,1))$, the control $f \varepsilon$ insensitizes $\Phi$ along the solutions of (1) if, and only if, the corresponding solution $(\bar{y}, q)$ of the cascade system (6-7) satisfies

$$
\|q(0, x)\|_{0}<\varepsilon,\left\|q_{t}(0, x)\right\|_{-1}<\varepsilon
$$

Proof. A simple calculation yields

$$
\left.\frac{d}{d \tau_{0}} \Phi(y)\right|_{\tau_{0}=\tau_{1}=0}=\int_{0}^{T} \int_{\mathcal{O}} \bar{y} \theta d x d t
$$

where $\theta=\frac{d y}{d \tau_{0}}$ is the solution of the equation

$$
\begin{cases}\theta_{t t}-\Delta \theta=0 & \text { in } \mathbb{R} \times(0,1), \\ \theta=0 & \text { in } \mathbb{R} \times\{0,1\} \\ \theta(0, x)=z^{0}, \quad \theta_{t}(0, x)=0 & \text { in }(0,1)\end{cases}
$$

On the other hand, integrating by parts in account of the boundary and initial (final) conditions in (7) and (10) we get

$$
\int_{0}^{T} \int_{\mathcal{O}} \bar{y} \theta d x d t=\int_{0}^{T} \int_{0}^{1} \bar{y} \mathbf{1}_{\mathcal{O}} \theta d x d t=\int_{0}^{T} \int_{0}^{1}\left(q_{t t}-q_{x x}\right) \theta d x d t=-\int_{0}^{1} q_{t}(0, x) z^{0} d x
$$


and thus, equality (9) becomes

$$
\left.\frac{d}{d \tau_{0}} \Phi(y)\right|_{\tau_{0}=\tau_{1}=0}=-\int_{0}^{1} q_{t}(0, x) z^{0} d x .
$$

In the same way we can prove that

$$
\left.\frac{d}{d \tau_{1}} \Phi(y)\right|_{\tau_{0}=\tau_{1}=0}=\int_{0}^{1} q(0, x) z^{1} d x .
$$

Finally, since $z^{0}, z^{1}$ are arbitrary functions satisfying

$$
\left\|z^{0}\right\|_{1}=\left\|z^{1}\right\|_{0}=1
$$

from (11)-(12) the assertion of the proposition holds.

The next step is to characterize the approximate controllability property of Proposition 1 in terms of the adjoint system to (6-7):

$$
\begin{aligned}
& \begin{cases}p_{t t}-\Delta p=0 & \text { in } \mathbb{R} \times(0,1), \\
p=0 & \text { in } \mathbb{R} \times\{0,1\}, \\
p(0, x)=p^{0}, \quad p_{t}(0, x)=p^{1} & \text { in }(0,1),\end{cases} \\
& \begin{cases}z_{t t}-\Delta z=z \mathbf{1}_{\mathcal{O}} & \text { in } \mathbb{R} \times(0,1), \\
z=0 & \text { in } \mathbb{R} \times\{0,1\}, \\
z(T, x)=0, \quad z_{t}(T, x)=0 & \text { in }(0,1) .\end{cases}
\end{aligned}
$$

We assume $\left(p^{0}, p^{1}\right) \in H_{0}^{1}(0,1) \times L^{2}(0,1)$. Thus system (13-14) has a unique solution $(p, z)$, which satisfies

$$
p, z \in C\left([0, T], H_{0}^{1}(0,1)\right) \cap C^{1}\left([0, T], L^{2}(0,1)\right) .
$$

In particular, $z \in L^{2}([0, T] \times(0,1))$.

The following result is easily proved by multiplying (6) by $z$ and integrating by parts.

Proposition 2 The following duality identity for the solutions of (6-7), (13-14) holds for every $f \in L^{2}(\mathbb{R} \times(0,1)),\left(y^{0}, y^{1}\right),\left(p^{0}, p^{1}\right) \in H_{0}^{1}(0,1) \times L^{2}(0,1)$

$$
\int_{[0, T] \times \omega} f z d x d t+\int_{[0, T] \times(0,1)} \xi z d x d t=\int_{0}^{1}\left(y^{0} z_{t}(0, x)-y^{1} z(0, x)\right) d x+\int_{0}^{1}\left(p^{0} q_{t}(0, x)-p^{1} q(0, x)\right) d x .
$$

Proposition 3 The set $\mathcal{B}=\left\{\left(q(0,),. q_{t}(0,).\right): f \in L^{2}(\mathbb{R} \times(0,1))\right\}$ is dense in $L^{2}(0,1) \times H^{-1}(0,1)$, and then the approximate controllability property (8) holds, if and only if the following unique continuation property is true for every $\left(p^{0}, p^{1}\right) \in H_{0}^{1}(0,1) \times L^{2}(0,1)$

$$
z \equiv 0 \text { in }[0, T] \times \omega \text { implies } z \equiv p \equiv 0 \quad \text { in }[0, T] \times(0,1) .
$$

Proof. Assume that $\left(p^{0}, p^{1}\right) \in H_{0}^{1}(0,1) \times L^{2}(0,1)$ satisfies

$$
\int_{0}^{1}\left(p^{0} q_{t}(0, x)-p^{1} q(0, x)\right) d x=0
$$

for every $f \in L^{2}(\mathbb{R} \times(0,1))$ and $\left(y^{0}, y^{1}\right) \in H_{0}^{1}(0,1) \times L^{2}(0,1)$. Then, in view of (15),

$$
\int_{[0, T] \times \omega} f z d x d t=-\int_{[0, T] \times(0,1)} \xi z d x d t+\int_{0}^{1}\left(y^{0} z_{t}(0, x)-y^{1} z(0, x)\right) d x .
$$


But the right hand term of this equality does not depend on $f$, so choosing $f=0$ it follows

$$
\int_{[0, T] \times \omega} f z d x d t=0 \quad \text { for every } f \in L^{2}(\mathbb{R} \times(0,1)),
$$

and then, since $z \in L^{2}([0, T] \times \omega)$, it follows that $z=0$ in $[0, T] \times \omega$. Next, the unique continuation property implies $p \equiv 0$ in $[0, T] \times(0,1)$, and hence $p^{0}=p^{1}=0$. Therefore, from the Hahn-Banach theorem it follows that $\mathcal{B}$ is dense in $L^{2}(0,1) \times H^{-1}(0,1)$.

For the converse assertion, assume that there exists $\left(p^{0}, p^{1}\right) \neq(0,0)$ such that $z \equiv 0$ in $[0, T] \times \omega$, and pick $\left(y^{0}, y^{1}\right)$ such that

$$
\int_{[0, T] \times(0,1)} \xi z d x d t=\int_{0}^{1}\left(y^{0} z_{t}(0, x)-y^{1} z(0, x)\right) d x .
$$

Then, from (15) we obtain

$$
\int_{0}^{1}\left(p^{0} q_{t}(0, x)-p^{1} q(0, x)\right) d x=0,
$$

and this means that $\mathcal{B}$ is not dense in $L^{2}(0,1) \times H^{-1}(0,1)$.

Gathering results of propositions 2 and 3 we obtain

Proposition 4 If the solutions of (13-14) satisfy the unique continuation property (16) then, for every $\varepsilon>0, \xi \in L^{2}(\mathbb{R} \times(0,1))$ and $\left(y^{0}, y^{1}\right) \in H_{0}^{1}(0,1) \times L^{2}(0,1)$ there exists a control $f^{\varepsilon} \in L^{2}(\mathbb{R} \times(0,1))$ such that along the corresponding solution $y^{\varepsilon}$ of system (1) the functional $\Phi$ verifies (5).

Remark 5 When $\omega \cap \mathcal{O} \neq \varnothing$ the unique continuation property (16) is reduced to a unique continuation property for the usual wave equation. Indeed, if $z \equiv 0$ in $[0, T] \times(\omega \cap \mathcal{O})$ so is $p$ in view of equation (32). Therefore, if $[0, T] \times(\omega \cap \mathcal{O})$ is an appropriate unique continuation region for the wave equation (that is, if the solution vanishes in $[0, T] \times(\omega \cap \mathcal{O})$ then it vanishes in $[0, T] \times(0,1))$, then $p \equiv 0$ in $[0, T] \times(0,1)$ and thus $z \equiv 0$ in $[0, T] \times(0,1)$. The same occurs in the case of heat equations considered in the literature.

Note that for the wave equation in $\mathbb{R}^{n}$ the unique continuation property from a region $[0, T] \times U$ depends on both $T$ and the geometry of $U$. For the $1-d$ wave equation considered here, unique continuation from a region $\left[t_{1}, t_{2}\right] \times U$ holds whenever $t_{2}-t_{1} \geq 2$ (twice the length of the interval $(0,1))$.

When $\omega \cap \mathcal{O}=\varnothing$ such arguments cannot be applied, and the problem becomes more difficult.

\subsection{Unique continuation results}

In the this subsection we show that the unique continuation property (16) is true for sufficiently large $T$ for arbitrarily chosen $\omega$ and $\mathcal{O}$, independently of whether their intersection is void or not. Indeed,

Proposition 6 Let $\omega, \mathcal{O}$ be non-empty open subsets of $[0,1]$ and $T \geq 4$. Then, the unique continuation property (16) holds for the solutions of system (13-14).

As a consequence of propositions 4 and 6 we obtain the existence of $\varepsilon$-insensitizing controls:

Theorem 7 Let $\omega, \mathcal{O}$ be non-empty open subsets of $[0,1]$ and $T \geq 4$. Then, for every $\varepsilon>0$, $\xi \in L^{2}(\mathbb{R} \times(0,1))$ and $\left(y^{0}, y^{1}\right) \in H_{0}^{1}(0,1) \times L^{2}(0,1)$ there exists a control $f^{\varepsilon} \in L^{2}(\mathbb{R} \times(0,1))$ that $\varepsilon$-insensitizes functional $\Phi$ along the solutions of (1). 
Proposition 6 is an immediate consequence of the following observability inequality:

Proposition 8 Let $\omega, \mathcal{O}$ be non-empty open subsets of $[0,1]$ and $T \geq 4$. Then, there exists a positive constant $C$, such that for every solution of (13-14),

$$
C\left\|\left(p^{0}, p^{1}\right)\right\|_{1,0}^{2} \int_{0}^{T} \int_{\omega} z^{2} d t d x \geq\left\|\left(p^{0}, p^{1}\right)\right\|_{0,-1}^{4} .
$$

Proof. We use in an essential way the fact that the first equation in (13) is the $1-d$ linear homogeneous wave equation

$$
\begin{cases}p_{t t}-\Delta p=0 & \text { in } \mathbb{R} \times(0,1), \\ p=0 & \text { in } \mathbb{R} \times\{0,1\}, \\ p(0, x)=p^{0}, \quad p_{t}(0, x)=p^{1} & \text { in }(0,1) .\end{cases}
$$

Let us recall some known facts we use in the proof. The solutions of (17) are periodic in time with period equal to 2 , what may be easily obtained, e.g., from the fact that the solutions are of the form

$$
p(t, x)=g(t+x)-g(t-x),
$$

with $g$ being a 2-periodic function.

Note that the derivatives $p_{x}$ and $p_{t}$ have the same periodicity property.

Moreover, from representation formula (18) one can prove the following well known unique continuation property for the solutions of (17): if $t^{*}-t_{*} \geq 2$ then any of the equalities $p=0$ in $\left[t_{*}, t^{*}\right] \times U$, or $p(t, 1)=0$ in $\left[t_{*}, t^{*}\right]$ or $p_{x}(t, 1)=0$ in $\left[t_{*}, t^{*}\right]$ implies $p=0$ in $\left[t_{*}, t^{*}\right] \times(0,1)$. This fact may be quantified by means of observability inequalities: There exist positive constants $C_{1}, C_{2}$ such that for every solution $p$ of (17)

$$
\begin{gathered}
C_{1} \int_{t_{*}}^{t^{*}} \int_{U} p^{2} d x d t \geq\left\|\left(p^{0}, p^{1}\right)\right\|_{0,-1}^{2}, \\
C_{2} \int_{t_{*}}^{t^{*}} p_{x}^{2}(t, 1) d t \geq\left\|\left(p^{0}, p^{1}\right)\right\|_{1,0}^{2} .
\end{gathered}
$$

Besides, there exist constants $C_{1}, C_{2}>0$ such that for every $\mu \in \mathbb{R}$

$$
\begin{gathered}
C_{1}\left\|\left(p^{0}, p^{1}\right)\right\|_{1,0} \leq\left\|\left(p(\mu, .), p_{t}(\mu, .)\right)\right\|_{1,0} \leq C_{2}\left\|\left(p^{0}, p^{1}\right)\right\|_{1,0} \\
C_{1}\left\|\left(p^{0}, p^{1}\right)\right\|_{0,-1} \leq\left\|\left(p(\mu, .), p_{t}(\mu, .)\right)\right\|_{0,-1} \leq C_{2}\left\|\left(p^{0}, p^{1}\right)\right\|_{0,-1} .
\end{gathered}
$$

Now we proceed to the proof of the proposition. Let $T_{0}$ be the smallest positive number such that $T-T_{0} \in 2 \mathbb{Z}$. The inequality stated in proposition is obtained from the following two inequalities:

$$
\begin{aligned}
C_{1} \int_{0}^{T} \int_{\omega} z^{2} d t d x & \geq\left\|\left(z\left(T_{0}\right), z_{t}\left(T_{0}\right)\right)\right\|_{0,-1}^{2} \\
C_{2}\left\|\left(z\left(T_{0}\right), z_{t}\left(T_{0}\right)\right)\right\|_{0,-1}\left\|\left(p^{0}, p^{1}\right)\right\|_{1,0} & \geq\left\|\left(p^{0}, p^{1}\right)\right\|_{0,-1}^{2},
\end{aligned}
$$

which hold for every solution $(p, z)$ of (13-14) with positive constants $C_{1}, C_{2}$ independent of $(p, z)$. 
In order to prove inequality (23) we define the linear operator $\ell$ acting on a function $u(t)$ by

$$
(\ell u)(t)=u(t-1)-u(t+1) .
$$

Note that $\ell$ is continuos from $L^{2}(\alpha, \beta)$ to $L^{2}(\alpha-1, \beta+1)$.

Further, for the solution $z$ of (14) we denote

$$
\psi(t, x)=(\ell z)(t, x)=z(t+1, x)-z(t-1, x) .
$$

The continuity of $\ell$ implies

$$
C \int_{0}^{T} \int_{\omega} z^{2} d x d t \geq \int_{1}^{T-1} \int_{\omega} \psi^{2} d x d t
$$

for some positive constant $C$ independent of $z$.

On the other hand, in view of the time periodicity of $p$, it follows that $\psi$ is a solution of the equation

$$
\begin{cases}\psi_{t t}-\Delta \psi=0 & \text { in } \mathbb{R} \times(0,1) \\ \psi=0 & \text { in } \mathbb{R} \times\{0,1\}\end{cases}
$$

and then, since $(T-1)-1=T-2 \geq 2$, inequalities (19) and (22) yield

$$
C \int_{1}^{T-1} \int_{\omega} \psi^{2} d x d t \geq\left\|\left(\psi(\mu, .), \psi_{t}(\mu, .)\right)\right\|_{0,-1}^{2},
$$

for every $\mu \in \mathbb{R}$.

Now denote $n=\left(T-T_{0}\right) / 2$ and observe that

$$
\begin{aligned}
\left\|z(T)-z\left(T_{0}\right)\right\|^{2} & =\left\|\sum_{k=0}^{n-1}(z(T-2 k)-z(T-2 k-2))\right\|^{2} \\
& \leq\left(\sum_{k=0}^{n-1}\|z(T-2 k)-z(T-2 k-2)\|\right)^{2} \\
& \leq n \sum_{k=0}^{n-1}\|z(T-2 k)-z(T-2 k-2)\|^{2} \\
& =n \sum_{k=0}^{n-1}\|\psi(T-2 k-1)\|^{2} .
\end{aligned}
$$

Thus, from inequality (26) we get

$$
\left\|z(T)-z\left(T_{0}\right)\right\|_{0}^{2} \leq n^{2} C \int_{1}^{T-1} \int_{\omega} \psi^{2} d x d t .
$$

Exactly in the same way it follows

$$
\left\|z_{t}(T)-z_{t}\left(T_{0}\right)\right\|_{-1}^{2} \leq n^{2} C \int_{1}^{T-1} \int_{\omega} \psi^{2} d x d t .
$$

From the last two inequalities, the fact that $z(T, x)=z_{t}(T, x)=0$ and (25) we obtain inequality (23). 
To prove the inequality (24) we multiply equation (14) by $p$ and integrate over $\left[T_{0}, T\right] \times(0,1)$. After integration by parts it follows

$$
\int_{T_{0}}^{T} \int_{\mathcal{O}} p^{2} d x d t=\int_{0}^{1}\left(z_{t}\left(T_{0}\right) p\left(T_{0}\right)-z\left(T_{0}\right) p_{t}\left(T_{0}\right)\right) d x .
$$

Now observe that, on one hand

$$
\int_{0}^{1}\left(z_{t}\left(T_{0}\right) p\left(T_{0}\right)-z\left(T_{0}\right) p_{t}\left(T_{0}\right)\right) d x \leq\left\|\left(z\left(T_{0}\right), z_{t}\left(T_{0}\right)\right)\right\|_{0,-1}\left\|\left(p\left(T_{0}\right), p_{t}\left(T_{0}\right)\right)\right\|_{1,0},
$$

and, on the other hand, from inequalities (19) and (22)

$$
\int_{T_{0}}^{T} \int_{\mathcal{O}} p^{2} d x d t \geq C\left\|\left(p^{0}, p^{1}\right)\right\|_{0,-1}^{2}
$$

Thus, from (27) inequality (24) is obtained.

Remark 9 Although the observability inequality provided by Proposition 8 allows us to ensure the appropriate unique continuation property and then, the approximate controllability of system (6-7), it is not good enough to guarantee the exact controllability of that system, since it do not allow to estimate a norm of $\left(p^{0}, p^{1}\right)$ in terms of the observed quantity $\int_{0}^{T} \int_{\omega} z^{2} d t d x$.

\section{Boundary control}

In this section we consider the boundary controlled system (2). We follow the same scheme as in the previous section. The main difference consists in that in this case we are able to prove the existence of (exact) insensitizing controls.

\subsection{Reduction to a controllability problem}

The proofs of the assertions given below use essentially the same arguments as in Section 2.

Consider the following controlled cascade system of wave equations

$$
\begin{aligned}
& \begin{cases}\bar{y}_{t t}-\Delta \bar{y}=\xi & \text { in } \mathbb{R} \times(0,1), \\
\bar{y}(t, 0)=0, \quad \bar{y}(t, 1)=g(t) & \text { in } \mathbb{R}, \\
\bar{y}(0, x)=y^{0}, \quad \bar{y}_{t}(0, x)=y^{1} & \text { in }(0,1),\end{cases} \\
& \begin{cases}q_{t t}-\Delta q=\bar{y} \mathbf{1}_{\mathcal{O}} & \text { in } \mathbb{R} \times(0,1), \\
q(t, 0)=0, \quad q(t, 1)=\bar{y}(t) & \text { in } \mathbb{R}, \\
q(T, x)=0, \quad q_{t}(T, x)=0 & \text { in }(0,1) .\end{cases}
\end{aligned}
$$

Proposition 10 Given $y^{0} \in H_{0}^{1}(0,1), y^{1} \in L^{2}(0,1)$ and $\xi \in L^{2}(\mathbb{R} \times(0,1))$, the control $f$ insensitizes $\Phi$ along the solutions of (2) if, and only if, the corresponding solution $(\bar{y}, q)$ of the cascade system (28-29) satisfies

$$
q(0, x)=q_{t}(0, x)=0 .
$$


The latter exact controllability property may be characterized with the help of the adjoint cascade system

$$
\begin{aligned}
& \begin{cases}p_{t t}-\Delta p=0 & \text { in } \mathbb{R} \times(0,1), \\
p=0 & \text { in } \mathbb{R} \times\{0,1\}, \\
p(0, x)=p^{0}, \quad p_{t}(0, x)=p^{1} & \text { in }(0,1),\end{cases} \\
& \begin{cases}z_{t t}-\Delta z=p 1_{\mathcal{O}} & \text { in } \mathbb{R} \times(0,1), \\
z=0 & \text { in } \mathbb{R} \times\{0,1\}, \\
z(T, x)=0, \quad z_{t}(T, x)=0 & \text { in }(0,1) .\end{cases}
\end{aligned}
$$

It holds

Proposition 11 Given $\left(y^{0}, y^{1}\right) \in H_{0}^{1}(0,1) \times L^{2}(0,1)$ and $g \in L^{2}(\mathbb{R})$, the solution $(\bar{y}, q)$ of (28-29) satisfies (30) if and only if, the following duality identity holds for every $\left(p^{0}, p^{1}\right) \in$ $L^{2}(0,1) \times H^{-1}(0,1)$

$$
\int_{0}^{T} g(t) z_{x}(1, t) d t+\int_{0}^{T} \int_{0}^{1} \xi z d x d t=\int_{0}^{1}\left(y^{0} z_{t}(0, x)-y^{1} z(0, x)\right) d x .
$$

\subsection{Observability inequalities}

Proposition 12 Let $\mathcal{O}$ be a non-empty open subset of $[0,1]$ and $T \geq 4$. Then, there exists a positive constant $C$, such that for every solution of (31-32),

$$
C \int_{0}^{T} z_{x}^{2}(1, t) d t \geq\left\|\left(p^{0}, p^{1}\right)\right\|_{0,-1}^{2} .
$$

Proof. The inequality given in the proposition is a consequence of the fact that there exist positive constants $C_{1}, C_{2}$, such that for every solution of (31-32),

$$
\begin{aligned}
C_{1} \int_{0}^{T} z_{x}^{2}(1, t) d t & \geq\left\|\left(z\left(T_{0}\right), z_{t}\left(T_{0}\right)\right)\right\|_{1,0}^{2}, \\
C_{2}\left\|\left(z\left(T_{0}\right), z_{t}\left(T_{0}\right)\right)\right\|_{1,0} & \geq\left\|\left(p^{0}, p^{1}\right)\right\|_{0,-1},
\end{aligned}
$$

where $T_{0}$ is the smallest positive number such that $T-T_{0} \in 2 \mathbb{Z}$.

These inequalities are proved using the same arguments as in Proposition 8.

\subsection{Existence of insensitizing controls}

Using Proposition 12 we can prove the following

Theorem 13 Let $\mathcal{O}$ be a non-empty open subset of $[0,1]$ and $T \geq 4$. Then, for every $\xi \in$ $L^{2}(\mathbb{R} \times(0,1))$ and $\left(y^{0}, y^{1}\right) \in H_{0}^{1}(0,1) \times L^{2}(0,1)$ there exists a control $g \in L^{2}(\mathbb{R} \times(0,1))$ that insensitizes $\Phi$ along the solutions of (2).

Proof. Consider the quadratic functional $J: L^{2}(0,1) \times H^{-1}(0,1) \rightarrow \mathbb{R}$ defined by

$$
J\left(p^{0}, p^{1}\right)=\int_{0}^{T} z_{x}^{2}(1, t) d t+\int_{0}^{T} \int_{0}^{1} \xi z d x d t-\int_{0}^{1}\left(y^{0} z_{t}(0, x)-y^{1} z(0, x)\right) d x,
$$

where $z$ is the corresponding solution of $(32)$. Let $\left(\hat{p}^{0}, \hat{p}^{1}\right)$ be the minimizer of $J$ provided by Proposition 14 (see below):

$$
J\left(\hat{p}^{0}, \hat{p}^{1}\right)=\min \left\{J\left(p^{0}, p^{1}\right):\left(p^{0}, p^{1}\right) \in L^{2}(0,1) \times H^{-1}(0,1)\right\} .
$$


Then, for every $\left(p^{0}, p^{1}\right) \in L^{2}(0,1) \times H^{-1}(0,1)$ the function $\phi(h)=J\left(\hat{p}^{0}+h p^{0}, \hat{p}^{1}+h p^{1}\right)$ has a minimum at $h=0$ and thus

$$
0=\phi^{\prime}(0)=\int_{0}^{T} \hat{z}_{x}(1, t) z_{x}(1, t) d t+\int_{0}^{T} \int_{0}^{1} \xi \hat{z} d x d t-\int_{0}^{1}\left(y^{0} \hat{z}_{t}(0, x)-y^{1} \hat{z}(0, x)\right) d x
$$

where $\hat{z}$ is the solution of (31-32) with initial data $\left(\hat{p}^{0}, \hat{p}^{1}\right)$. Consequently, if we choose $g(t)=$ $\hat{z}_{x}(1, t)$ equality (33) is verified. Therefore, the control $g(t)=\hat{z}_{x}(1, t)$ insensitizes $\Phi$.

Proposition 14 The functional $J$ has a minimizer $\left(\hat{p}^{0}, \hat{p}^{1}\right) \in L^{2}(0,1) \times H^{-1}(0,1)$.

Proof. $J$ in continuous and convex. So, to guarantee the existence of a minimizer it is sufficient to prove that $J$ is coercive, i.e., $J \rightarrow \infty$ as $\left\|\left(p^{0}, p^{1}\right)\right\|_{0,-1} \rightarrow \infty$.

Let us note first that $z$ verifies

$$
\begin{gathered}
\int_{0}^{T} \int_{0}^{1} z^{2} d x d t \leq C \int_{0}^{T} \int_{\mathcal{O}} p^{2} d x d t \leq C\left\|\left(p^{0}, p^{1}\right)\right\|_{0,-1}^{2} \\
\left\|\left(z(0), z_{t}(0)\right)\right\|_{1,0} \leq C \int_{0}^{T} \int_{\mathcal{O}} p^{2} d x d t \leq C\left\|\left(p^{0}, p^{1}\right)\right\|_{0,-1}^{2} .
\end{gathered}
$$

Consequently,

$$
\begin{aligned}
& \left|\int_{0}^{T} \int_{0}^{1} \xi z d x d t\right| \leq\left(\int_{0}^{T} \int_{0}^{1} \xi^{2} d x d t\right)^{\frac{1}{2}}\left(\int_{0}^{T} \int_{0}^{1} z^{2} d x d t\right)^{\frac{1}{2}} \\
& \leq C\|\xi\|_{L^{2}((0, T) \times(0,1))}\left\|\left(p^{0}, p^{1}\right)\right\|_{0,-1}, \\
& \left|\int_{0}^{1}\left(y^{0} z_{t}(0)-y^{1} z(0, x)\right) d x\right| \leq\left\|\left(y^{0}, y^{1}\right)\right\|_{0,-1}\left\|\left(z(0), z_{t}(0)\right)\right\|_{1,0} \\
& \leq C\left\|\left(y^{0}, y^{1}\right)\right\|_{0,-1}\left\|\left(p^{0}, p^{1}\right)\right\|_{0,-1} .
\end{aligned}
$$

From Proposition 12, (34) and (35) we get

$$
\left|J\left(p^{0}, p^{1}\right)\right| \geq\left\|\left(p^{0}, p^{1}\right)\right\|_{0,-1}\left(\left\|\left(p^{0}, p^{1}\right)\right\|_{0,-1}-C\|\xi\|_{L^{2}((0, T) \times(0,1))}-\left\|\left(y^{0}, y^{1}\right)\right\|_{0,-1}\right)
$$

and this implies that $J \rightarrow \infty$ as $\left\|\left(p^{0}, p^{1}\right)\right\|_{0,-1} \rightarrow \infty$, and hence the assertion of the proposition is proved.

\section{Final remarks}

The condition that the observation time be large $(T \geq 4)$, which does not appear in previous papers in the context of the heat equations, is natural for the wave equation due to the finite speed of propagation of its solutions. Indeed, it is possible to prove that, for every $T<2$ one can choose an observation region $\mathcal{O}$ such that certain non-trivial solution of (17) satisfies $p=0$ in $[0, T] \times \mathcal{O}$, and then, $z=0$ in $[0, T] \times(0,1)$. This means that the unique continuation property is not verified. One may expect however the property to be true for $0<T<4$ and in fact, we do not know whether the property is true in this case or not. Nevertheless, the condition $T \geq 4$ (twice the sum of the lengths of the strings) appears to be natural in the context of coupled 
wave equations. Several examples of this situation are given in [5]. Note however, that for every particular choice of the observation interval $\mathcal{O}$ the minimal observation time to guarantee the unique continuation property is strictly smaller than 4 .

Of course, it would be of interest to study the insensitizing properties of the controlled systems for other choice of the observation functionals, in particular,

$$
\Phi(y)=\frac{1}{2} \int_{0}^{T} \int_{\mathcal{O}}\left(y_{t}^{2}+y_{x}^{2}\right) d x d t
$$

which may be understood as the mean value of the energy of the portion $\mathcal{O}$ of the string in a time interval of length $T$. This problem turns out to be more difficult, at least at the level of reducing it to a controllability property.

It is clear that the method we have used to prove the unique continuation do not apply to other $1-d$ equations, for which the time periodicity property is not valid. However, it does not mean that the results are not valid for those equations. We hope, that the existence of $\varepsilon$-insensitizing controls for non-intersecting observation and control regions is not restricted to the $1-d$ wave equation, and the example given in [10] suggests that this property is also true for the $1-d$ heat equation.

\section{References}

[1] O. Bodart, M. González-Burgos, and R. Pérez-García. Existence of insensitizing controls for a semilinear heat equation with a superlinear nonlinearity. Comm. Partial Differential Equations, 29(7-8):1017-1050, 2004.

[2] O. Bodart, M. González-Burgos, and R. Pérez-García. Insensitizing controls for a heat equation with a nonlinear term involving the state and the gradient. Nonlinear Anal., 57(5-6):687-711, 2004.

[3] Olivier Bodart and Caroline Fabre. Controls insensitizing the norm of the solution of a semilinear heat equation. J. Math. Anal. Appl., 195(3):658-683, 1995.

[4] Olivier Bodart, Manuel González-Burgos, and Rosario Pérez-García. A local result on insensitizing controls for a semilinear heat equation with nonlinear boundary Fourier conditions. SIAM J. Control Optim., 43(3):955-969 (electronic), 2004.

[5] René Dáger and Enrique Zuazua. Wave propagation, observation and control in $1-d$ flexible multi-structures, volume 50 of Mathématiques $\&$ Applications (Berlin) [Mathematics Es Applications]. Springer-Verlag, Berlin, 2006.

[6] Luz de Teresa. Controls insensitizing the norm of the solution of a semilinear heat equation in unbounded domains. ESAIM Control Optim. Calc. Var., 2:125-149 (electronic), 1997.

[7] Luz de Teresa. Insensitizing controls for a semilinear heat equation. Comm. Partial Differential Equations, 25(1-2):39-72, 2000.

[8] Enrique Fernández-Cara, Galina C. Garcia, and Axel Osses. Controls insensitizing the observation of a quasi-geostrophic ocean model. SIAM J. Control Optim., 43(5):1616-1639 (electronic), 2005. 
[9] J.-L. Lions. Quelques notions dans l'analyse et le contrôle de systèmes à données incomplètes. In Proceedings of the XIth Congress on Differential Equations and Applications/First Congress on Applied Mathematics (Spanish) (Málaga, 1989), pages 43-54, Málaga, 1990. Univ. Málaga.

[10] S. Micu, J. H. Ortega, and L. de Teresa. An example of $\epsilon$-insensitizing controls for the heat equation with no intersecting observation and control regions. Appl. Math. Lett., 17(8):927932, 2004 .

[11] Sorin Micu, Jaime H. Ortega, and Luz de Teresa. $\epsilon$-insensitizing controls for pointwise observations of the heat equation. Systems Control Lett., 51(5):407-415, 2004. 